\title{
Tratamento cirúrgico da epilepsia refratária: análise de 34 casos atendidos no Hospital das Clínicas da UFMG
}

\author{
Leandro Custódio do Amaral', Eduardo Jardel Portela², Marcelo Magaldi Ribeiro de \\ Oliveira ${ }^{3}$, Marilis Tissot Lara4, Sílvio Roberto de Sousa-Pereira², Liz Custódio Souza \\ Seabra ${ }^{5}$, Marcelo Gomes de Almeida ${ }^{6}$, Guilherme Marques Miranda de Menezes ${ }^{6}$
}

Hospital das Clínicas da Universidade Federal de Minas Gerais (UFMG), Belo Horizonte, MG, Fundação Benjamin Guimarães, Hospital da Baleia, Belo Horizonte, MG, Brasil.

\section{RESUMO}

Objetivo: Apresentar dados estatísticos referentes a uma série de casos submetidos ao tratamento cirúrgico da epilepsia refratária no Hospital das Clínicas da Universidade Federal de Minas Gerais (HC-UFMG), no período entre 23/11/2007 e 7/12/2010. Métodos: Estudo retrospectivo com análise de prontuários de 34 pacientes e classificação do controle de crises pós-operatório utilizando a Escala de Engel. Resultados: Dos pacientes, 70,5\% apresentaram início das crises antes dos 15 anos, sendo o tipo de crise mais comum as crises parciais complexas com generalização secundária, presente em $55 \%$ dos casos. Esclerose mesial temporal foi o diagnóstico de base em $79,4 \%$ dos pacientes. Houve complicações cirúrgicas em $23,5 \%$ dos casos, sendo as mais frequentes as alterações de campo visual (8,8\% dos casos). Conclusão: O controle de crises foi compatível com Engel menor ou igual a III em $64,7 \%$ dos casos. O tratamento cirúrgico revelou-se eficiente para melhorar o controle de crises em pacientes portadores de epilepsia refratária ao tratamento medicamentoso.

\section{PALAVRAS-CHAVE}

Epilepsia/cirurgia, epilepsia/complicações, epilepsia do lobo temporal, esclerose.

\section{ABSTRACT}

Surgical treatment of refractory epilepsy: analysis of 34 cases from the Hospital das Clínicas, Federal University of Minas Gerais

Objective: Presenting statistical data on a series of cases undergoing surgical treatment of epilepsy at the Hospital das Clínicas, Universidade Federal de Minas Gerais (HC-UFMG) in the period between 11/23/2007 and 12/7/2010. Methods: Retrospective analysis with charts of 34 patients and classification of seizure control postoperatively using the Engel Scale. Results: $70.5 \%$ of the patients had the onset of seizures before 15 years old and the most common type of seizure was complex partial seizure with secondary generalization, presented in $55 \%$ of cases. Mesial temporal sclerosis was the underlying diagnosis in $79.4 \%$ of patients. There were surgical complications in $23.5 \%$ of cases, the most frequent visual field changes (8.8\% of cases). Conclusion: Seizure control was consistent with Engel III or less in $64.7 \%$ of cases. Surgical treatment has proved effective in improving seizure control in patients with medically refractory epilepsy.

\section{KEYWORDS}

Epilepsy/surgery, epilepsy/complications, epilepsy temporal lobe, sclerosis.

Residente de Neurocirurgia da Fundação Benjamin Guimarães, Hospital da Baleia, Belo Horizonte, MG, Brasil.

2 Médico do Serviço de Neurologia do Hospital das Clínicas da Universidade Federal de Minas Gerais (UFMG), Belo Horizonte, MG, Brasil.

Professor adjunto do Departamento de Cirurgia da UFMG, Belo Horizonte, MG, Brasil.

Preceptora da Residência Médica de Neuropediatria do Hospital das Clínicas da UFMG, Belo Horizonte, MG, Brasil.

5 Residente de Pediatria do Hospital das Clínicas da UFMG, Belo Horizonte, MG, Brasil.

6 Acadêmico de Medicina da Faculdade de Medicina da UFMG, Belo Horizonte, MG, Brasil. 


\section{Introdução}

A epilepsia afeta cerca de $0,5 \%$ a $1 \%$ da população mundial, ${ }^{1}$ acometendo mais de 50 milhões de pessoas. ${ }^{2}$ Dessas, cerca de $25 \%$ a $30 \%$ apresentam crises epiléticas refratárias à terapia medicamentosa. ${ }^{3}$ Boa parte desses pacientes apresenta crises parciais complexas consequentes à esclerose mesial temporal, constituindo o maior contingente passível de tratamento cirúrgico. ${ }^{2}$

As crises epilépticas mal controladas podem acarretar danos físicos, cognitivos, psicológicos e sociais para os pacientes. ${ }^{2,45}$ Por isso, a indicação cirúrgica criteriosa, ao permitir eficiente controle de crises, pode contribuir positivamente para a qualidade de vida dos pacientes com epilepsia refratária. ${ }^{2}$

\section{Objetivos}

Obter dados estatísticos referentes a uma série de casos de pacientes submetidos ao tratamento cirúrgico para tratamento de epilepsia refratária no Hospital das Clínicas da Universidade Federal de Minas Gerais, no período entre 23/11/2007 e 7/12/2010.

Analisar os dados apurados de forma a permitir comparação dos resultados obtidos no HC-UFMG com aqueles constantes na literatura e viabilizar a implementação de melhorias no atendimento aos pacientes.

\section{Materiais e métodos}

Estudo retrospectivo com análise de prontuários de 34 pacientes submetidos à neurocirurgia para tratamento de epilepsia no HC-UFMG. Para avaliar e comparar os resultados cirúrgicos com relação ao controle de crises, foi utilizada a escala de Engel $^{6}$ (Tabela 1).

Tabela 1 - Escala de Engel

I. Livre de crises incapacitantes

II. Crises incapacitantes raras

III. Melhora evidente

IV. Sem melhora evidente

Adaptada de: Engel J Jr. Surgery for seizures. N Engl J Med. 1996;334(10):647-52.

\section{Resultados}

Dos 34 pacientes incluídos no estudo, 20 (58,8\%) eram do sexo feminino e 16 (47\%) deles apresentavam história familiar positiva para epilepsia.
No que se refere à idade de início das crises epilépticas, nota-se que a imensa maioria começou a apresentar crises na infância ou na adolescência. Vinte e quatro pacientes $(70,5 \%)$ tiveram a primeira crise antes dos 15 anos e 10 , entre 16 e 50 anos. Portanto, nenhum deles teve a primeira crise epiléptica na idade adulta avançada ou na terceira idade.

Quanto ao tipo de crise epiléptica, 26 pacientes (76,5\%) apresentaram crises parciais complexas, sendo esse o tipo mais comum. Porém, 19 pacientes (55,8\%) evoluíram com generalização secundária dessas crises. Em um número menor de pacientes, cinco deles (14,7\%), houve apenas crises tônico-clônicas generalizadas.

Dos pacientes incluídos no estudo, nove pacientes (26,5\%) faziam uso de monoterapia, $13(38,2 \%)$, de politerapia com duas medicações, $11(32,4 \%)$, de politerapia com três medicações e um $(2,9 \%)$ usava cinco fármacos.

Em relação ao tempo de tratamento farmacológico prévio, constatou-se que 20 pacientes $(58,8 \%)$ estavam em tratamento há mais de 20 anos. Uma minoria dos pacientes $(32,5 \%)$ estava em uso de terapia farmacológica por período entre 5 e 20 anos.

O diagnóstico etiológico mais comum é a esclerose mesial temporal, presente em 28 pacientes $(82,4 \%)$. Desses, 12 pacientes $(42,9 \%)$ apresentavam lesão do hipocampo esquerdo e $14(50 \%)$, do hipocampo direito; $2(7,1 \%)$ tinham lesão bilateral.

Apenas quatro pacientes (11,76\%) apresentaram queixas cognitivas pré-operatórias. Contudo, durante avaliação neuropsicológica, 24 pacientes $(70,6 \%)$ foram diagnosticados com algum déficit de memória. Entre esses, um paciente $(4,16 \%)$ tinha apenas déficit de memória verbal, 17 (70,84\%), apenas déficit de memória não verbal e seis (25\%), déficit de ambas.

A maior parte dos pacientes (70,6\%) foi submetida ao procedimento cirúrgico entre 16 e 50 anos. Outros cinco pacientes $(14,7 \%)$ foram operados com mais de 50 anos e apenas quatro $(11,76 \%)$, em idade inferior a 15 anos.

A grande maioria dos pacientes, 26 deles $(76,6 \%)$, foi submetida à amígdalo-hipocampectomia, sendo essa a cirurgia mais comumente realizada. Um paciente (2,9\%) foi submetido à lobectomia temporal padrão, quatro $(11,8 \%)$, à lesionectomia, dois $(5,8 \%)$, à calosotomia e um (2,9\%), à técnica indeterminada.

Houve complicações pós-operatórias em oito pacientes (23,5\%). Em dois deles (25\%) a complicação foi de origem infecciosa; outros dois (25\%) apresentaram déficit motor e três $(37,5 \%)$ tiveram alterações de campo visual. Em um paciente $(12,5 \%)$ a complicação não foi determinada.

Cinquenta por cento dos pacientes ficaram livres de crises epilépticas incapacitantes após a cirurgia (Engel I); um paciente (2,9\%) manteve apenas crises incapacitantes raras (Engel II); nove pacientes (26,5\%) obtiveram melhora significativa das crises com a cirurgia (Engel 
III); três pacientes $(8,8 \%)$ não puderam ser classificados de acordo com a escala de Engel; e apenas quatro pacientes $(11,8 \%)$ evoluíram sem melhora significativa dos sintomas (Engel IV).

\section{Discussão}

Uma grande variedade de estratégias cirúrgicas tem sido utilizada no tratamento das epilepsias refratárias à terapêutica medicamentosa; a lobectomia temporal é o procedimento mais utilizado nesses casos. ${ }^{2}$ Uma variedade de métodos é utilizada para planejar a extensão da ressecção e as diferentes abordagens cirúrgicas, mas o resultado no controle de crises tem sido excelente nos pacientes com epilepsia de lobo temporal que foram adequadamente selecionados. Aproximadamente, $60 \%$ a $80 \%$ desses pacientes ficam livres das crises epilépticas no longo $\mathrm{prazo}^{7}$ quando comparados a menos de $10 \%$ daqueles que continuaram a ser manejados conservadoramente. Já as epilepsias cujos focos se localizam fora do lobo temporal representam maior desafio cirúrgico. Esse grupo heterogêneo de doenças exige avaliação pré-operatória mais extensa e apresenta pior resultado no que se refere ao controle de crises. ${ }^{8}$ Dessa forma, mesmo quando os focos epileptiformes são localizados em áreas não eloquentes, apenas $50 \%$ a $60 \%$ dos pacientes submetidos a ressecções extratemporais ficam livres de crises. O pior resultado no controle de crises do grupo estudado em relação ao observado na literatura pode ser explicado pela heterogeneidade do grupo estudado, já que $17,6 \%$ dos pacientes apresentavam epilepsia extratemporal, pela perda de seguimento de três pacientes $(8,8 \%$ do total) que não puderam ser classificados segundo a escala de Engel e também pelo curto tempo de segmento de alguns pacientes, visto que alguns deles foram seguidos apenas por poucos meses. Vale destacar que novas formas de avaliar os resultados cirúrgicos têm sido desenvolvidas, uma vez que mesmo pacientes Engel IV podem apresentar uma redução de até $70 \%$ na frequência de crises, porém sem melhora expressiva na qualidade de vida, a qual pode ser prejudicada mesmo por um número pequeno de crises por ano.

No que se refere às complicações pós-operatórias, as taxas classicamente descritas têm sido algo entre $10 \%$ e $20 \%$, ${ }^{1,2}$ porém esses valores são bastante variáveis conforme a frequência de cada procedimento cirúrgico. Dessa forma, a taxa de complicações pós-operatórias do estudo em questão foi apenas discretamente superior a esperada. Contudo, no que se refere às complicações infecciosas, elas foram completamente resolvidas, e os déficits motores evoluíram com melhora ao menos parcial. As complicações visuais foram principalmente pequenos defeitos de campo visual, ou seja, apresentaram baixa morbidade.

\section{Conclusão}

O tratamento cirúrgico das epilepsias refratárias ao tratamento medicamentoso revelou-se eficiente na medida em que permitiu melhora importante no controle de crises com baixa morbimortalidade, tendo em vista a reversibilidade de várias complicações pós-operatórias.

\section{Conflito de interesses}

Os autores declaram não haver conflito de interesses.

\section{Referências}

1. Meneses MS, Rocha SB, Kowacs PA, Andrade NO, Santos HL, Narata AP, et al. Surgical treatment of temporal lobe epilepsy: a series of forty-three cases analysis. Arq Neuropsiquiatr. 2005;63(3A):618-24.

2. Alvarenga KG, Garcia GC, Ulhôa AC, Oliveira AO, Mendes MFSG, Cesarini IM, et al. Epilepsia refratária: a experiência do núcleo avançado de tratamento das epilepsias do Hospital Felício Rocho (NATE) no período de março de 2003 a dezembro de 2006. J Epilepsy Clin Neurophysiol. 2007;13(2):71-4.

3. Wieser HG, Ortega M, Friedman A, Yonekawa Y. Long-term seizures outcomes following amygdalectomy. J Neurosurg. 2003;98:751-63.

4. Garriga-Canut M, Schoenike B, Qazi R, Bergendah K, Daley TJ, Pfender RM, et al. 2-deoxy-D-glucose reduces epilepsy progression by NRSF-CtBP-dependent metabolic regulation of chromatin structure. Nat Neurosci. 2006;9(11):1382-7.

5. Levin R, Banks S, Berg B. Psychosocial dimensions of epilepsy: a review of the literature. Epilepsia. 1988;29(6):80516.

6. Engel JJ. Surgery for seizures. N Engl J Med. 1996; 334(10):647-52.

7. Bingaman W, Najm I. Standard temporal lobectomy. In: Winn HR, editor. Youmans neurological surgery. 6th ed. Philadelphia: Elsevier Saunders; 2011. p. 767-73.

8. Leiphart JW, Fried I. Surgery for extratemporal lobe epilepsy. In: Winn HR, editor. Youmans neurological surgery. 6th ed. Philadelphia: Elsevier Saunders; 2011. p. 754-66.

Endereço para correspondência

Leandro Custódio do Amaral

Rua Grão Pará, 638, ap. 600, Bairro Santa Efigênia

30150-341 - Belo Horizonte, MG, Brasil

Telefone: (31) 3214-0771

E-mail: leandroamaral_ns@hotmail.com 